\title{
Intimités et violences : l'intimité conjugale violente et la destruction de l'intimité personnelle
}

\author{
Intimidades e violências: a intimidade conjugal violenta e a destruição da privacidade \\ pessoal \\ Intimates and violence: a violent marriage intimacy and destruction of personal \\ privacy \\ Angélica Mateus Mora \\ Université François-Rabelais de Tours, France \\ UFR Lettres et Langues, Département d'Études hispaniques
}

\begin{abstract}
Résumé
L'écrivaine espagnole Mercedes Pinto publie dans l'exil en 1926 le récit littéraire Él. L'auteure décrit une relation conjugale très marquée par une asymétrie de pouvoir entre une épouse dominée et un mari paranoïaque, misanthrope et narcissique. En privilégiant la violence psychique, Luis Buñuel en fait, en 1952, l'objet d'une adaptation cinématographique. Ces deux récits, littéraire et cinématographique, de l'intimité conjugale violente, qui ne peuvent pas être dissociés du contexte social dans lequel ils ont été produits, ne se limitent pas à représenter la violence dans l'intime : ils décrivent également une forme d'intimité conjugale qui est en elle-même et par elle même violence. L'analyse de ces deux représentations de l'intimité conjugale violente permet de caractériser cette forme d'intimité du couple comme un type de relation dans lequel l'intimité se transforme en enfermement ou privation du lien social. Par ailleurs, en distinguant deux niveaux de l'intimité - l'intimité à deux et l'intimité personnelle- l'analyse permet d'établir la signification violente de la logique d'enferment qui, dans sa forme la plus radicale, aboutit à la destruction de l'intimité personnelle. Du patriarcat à la Féminitude: violence sexuelle et conflits de genre dans la prose romanesque de Calixthe Beyala.
\end{abstract}

Mots-clés: Intimité; Violences ; Femmes; Relation conjugale.

\begin{abstract}
Resumo
A escritora espanhola Mercedes Pinto publicou no exílio, em 1926, narrativa literária “Él”. A autora descreve um relacionamento conjugal por uma assimetria muito acentuada de poder entre uma esposa dominada e um marido paranoico, misantropo e narcisista. Focalizando a violência psíquica, Luis Buñuel, efetivamente, em 1952, objeto de uma adaptação para o cinema. Estas duas histórias, literária e cinematográfica, de intimidade conjugal violenta que não podem ser separadas do contexto social em que foram produzidas, não se limitam a representar a violência no íntimo: elas também descrevem uma forma de intimidade conjugal que é em si, por si mesmo violência. A análise dessas duas representações da intimidade conjugal violenta permite caracterizar esta forma de intimidade do casal como um tipo de relacionamento em que a intimidade se torna confinamento ou privação de laços sociais. Além disso, a distinção entre dois níveis de intimidade - a intimidade a dois e intimidade pessoal - a análise permite estabelecer o significado violento do bloqueio lógico que, na sua forma mais radical, leva a destruição da privacidade pessoal. Do patriarcado a Feminilidade: a violência sexual e os conflitos de gênero na prosa romântica Calixthe Beyala.

Palavras-chaves: Intimidade; Violência; Mulheres; Relação conjugal.
\end{abstract}

\begin{abstract}
The Spanish writer Mercedes Pinto published in exile in 1926, literary narrative "Él". The author describes a conjugal relationship for a very marked power asymmetry between a dominated wife and paranoid, misanthropic, narcissistic husband. Luis Buñuel, focusing on psychological violence, effectively, made a film adaptation, in 1952. These two stories, literary and cinematic of violent marital intimacy that cannot be separated from the social context in which they were produced, do not merely represent the violence in intimacy: they also describe a form of marital intimacy which is in itself and by itself violence. The analysis of these two representations of violent marital intimacy allows to characterize this form of the couple intimacy as a type of relationship in which intimacy becomes confinement or deprivation of social ties. Moreover, the distinction between two levels of intimacy - the intimacy with your partner and personal intimacy - the analysis allows to establish the violent meaning of the logical block that, in its most radical form, leads to destruction of personal privacy. From Patriarchy to Femininity: sexual violence and gender conflicts in romantic prose Calixthe Beyala.
\end{abstract}

Key-words: Intimacy; Violence; Women; Marital relationship.

\section{Introduction}

Nous aborderons le thème « intimités et violences » à partir d'un récit autobiographique de l'intimité : le récit littéraire Él («Lui »), publié en 1926 à
Montevideo par l'écrivaine espagnole Mercedes Pinto (1883-1976), et adapté au cinéma par Luis Buñuel en 1952. Mercedes Pinto avait quitté l'Espagne deux ans plus tôt, en 1924, après avoir été informée que le dictateur Primo de Rivera envisageait de la 
déporter dans la colonie africaine de Fernando Poo. La déportation aurait été liée à la lecture publique de son texte «El divorcio como medida higiénica 》 à l'Université Centrale de Madrid, en novembre 1923. Le film de Buñuel, qui reprend le titre Él (en français : " Tourments »), comporte selon le réalisateur « un certain nombre de détails vrais, empruntés à l'observation quotidienne, et aussi une bonne part d'invention». (Buñuel, 2006, p. 251). Bien que Pinto ne présente pas explicitement son texte comme le récit d'une période de sa vie, Él est considéré par l'ensemble de la critique comme un document autobiographique qui décrit « un enfer personnel » effectivement vécu et attesté par de nombreux documents (Cummings, 2006, p. 35).

Dans le texte littéraire, la narratrice raconte l'histoire de sa vie de couple avec son mari, qu'elle désigne uniquement par le pronom masculin «Él» dans l'ensemble du récit. À travers cette narratrice qui se maintient dans l'anonymat du début jusqu'à la fin, l'auteure décrit une relation conjugale très fortement marquée par une asymétrie de pouvoir, avec un mari dominateur et une épouse dominée. Ce rapport de domination est exacerbé par le psychisme particulier de l'époux, qui est présenté comme un être paranoïaque, misanthrope et narcissique. L'expérience d'assujettissement et de violence conjugale subi par la narratrice se prolonge une dizaine d'années. Le récit Él ne précise ni le temps ni la durée de l'expérience vécue : la narratrice se sert d'expressions du temps telles que « un día », " otra noche », " en aquella ocasión », « aquella tarde », sans jamais indiquer une date, une année ou une époque précise. En revanche, le récit autobiographique Ella (1934) fournit une série d'éléments qui nous aident à déterminer la durée de cette relation conjugale : par exemple, lorsque la narratrice raconte la mort de sa sœur, on apprend que la vie commune dure déjà depuis neuf ans : " Durante nueve años fui mandada como un siervo (...) nueve años en que mi salud magnifica permitía la resistencia a todas las tormentas...» (Pinto, 2011b, p. 271) ; plus loin, lors de la deuxième tentative de suicide de son époux : «...repasé mil veces en minutos los sucesos, los escándalos, los golpes, los tormentos de diez años...» (Pinto, 2011b, p. 280). D'après ces indications, la cohabitation conjugale n'a pas duré moins d'une dizaine d'années et s'achève par la fuite de la femme avec ses enfants loin du domicile conjugal, à la suite d'une grave crise de délire paranoïaque du mari, au cours de laquelle il tente de se suicider une deuxième fois. Dans l'adaptation cinématographique du récit, Buñuel conserve la structure de base du récit - l'intimité conjugale violente - tout en privilégiant la description de la violence psychique plutôt que celle de la violence corporelle - pour en faire ce que le réalisateur espagnol nomme le « portrait d'un paranoïaque » (Buñuel, 2006, p. 251).

Ces deux récits, littéraire et cinématographique, de l'intimité conjugale violente ne se limitent pas à représenter la violence dans l'intimité conjugale. À nos yeux, l'intérêt principal de ces deux œuvres réside dans leur description d'une forme d'intimité conjugale qui est en elle-même et par elle-même violence, celleci étant définie comme atteinte à l'intégrité de la personne, et comprenant dès lors la domination sur les personnes.

L'analyse de ces deux représentations de l'intimité conjugale violente permet de caractériser cette forme d'intimité du couple comme un type de relation dans lequel l'intimité se transforme en enfermement ou privation de lien social : l'intime est signifié ici comme le fermé au social, de telle manière que l'intimité du couple peut avoir le sens d'une « prison » pour l'élément dominé, en l'occurrence «Ella». Par ailleurs, en distinguant deux niveaux de l'intimité l'intimité à deux et l'intimité personnelle - l'analyse permet d'établir la signification violente de la logique de l'enfermement qui, dans sa forme sans doute la plus radicale, rendue manifeste par la pathologie psychique de « $E l$ », aboutit à la destruction de l'intimité personnelle, c'est-à-dire à la négation de la personne singulière en tant que source autonome de significations et de valeurs, définie par une expérience unique du monde et du temps.

\section{La construction de l'intimité du couple comme enfermement}

Dans ces deux œuvres l'approche de l'enfermement diffère: dans le récit Él de Mercedes Pinto, la narratrice présente une succession de souvenirs qui construisent progressivement les personnalités de Él et de Ella, ainsi que leur relation de couple ; le récit se présente comme un rappel douloureux d'une série d'épisodes retraçant l'assujettissement de Ella, mais ne fournit aucune indication sur la construction initiale de la relation conjugale : c'est seulement dans Ella, un nouveau récit écrit par Pinto en 1934, que le lecteur peut trouver des éléments permettant de comprendre l'installation de Ella dans une intimité conjugale violente.

En revanche, le film Él de Buñuel, et c'est là certainement l'un de ces apports au récit original de Pinto, montre en quelque sorte la totalité de l'histoire, du début de la relation jusqu'à sa dissolution. Au départ, Ella (Gloria, dans le film) apparaît comme une femme relativement libre et capable de prendre des décisions, y compris celle de rompre ses fiançailles avec Raúl afin d'épouser Él (Francisco, dans le film), son futur bourreau.

Peu à peu, Gloria perd cette capacité. Évitant le risque de simplification qui consisterait à montrer un pervers qui attrape une victime innocente, Buñuel montre que l'enfermement se construit précisément dans et par la relation du couple : il y a un certain consentement de Ella à la domination de Él. Au 
début, Gloria est précisément fascinée par l'attitude dominante de Francisco : « ton air de maîtrise, ton assurance (...) m'ont éblouie chez toi » lui dit-elle. Cet éblouissement, qui peut exprimer un besoin de protection et de sécurité, renvoie en même temps à une apparence : l'image sociale de Francisco est très positive : «J'ai été trompée (...), comme tous les autres (...) ; tout le monde l'aime et le respecte. Ils ne savent pas comment il est en réalité $\gg$ - dira un jour Gloria à Raúl (28 minutes 4 secondes).

Par touches successives, Buñuel montre la série de renoncements et d'acceptations de l'intolérable qui engagent peu à peu Gloria - par conformisme, par peur ou par pusillanimité - dans la construction d'une intimité-enfermement. Ainsi, lors de leur lune de miel, Francisco oblige Gloria à ne plus saluer une vieille connaissance qu'elle rencontre dans la rue; plus tard, il généralise l'interdiction à Gloria de parler avec des « étrangers », et considérera la mère de Gloria - son seul contact avec le monde extérieur, à un moment donné - comme une intruse.

Je n'aime pas que quelqu'un puisse te soutirer des choses relatives à notre vie intime »- déclare Francisco, dressant un mur entre la « vie intime» et les autres, perçus systématiquement comme «étrangers » ( «Il est humiliant de raconter notre vie à un étranger) (1 heure 12 minutes 8 secondes).

À partir d'un certain moment, Gloria se découvre littéralement enfermée : elle doit demander l'autorisation à Francisco pour rendre visite à sa mère, et la belle demeure du couple devient une sorte de cage dorée: l'intimité-enfermement est tout d'abord enfermement dans un espace physique. "J'ai vécu plusieurs mois sans qu'il me laissât voir personne » - confiera Gloria à Raúl, qu'elle retrouve par hasard dans une rue au cours d'une échappée désespérée. À l'intérieur même de cet espace clos, Gloria ne peut accéder à certains endroits qu'avec l'autorisation de Francisco.

Plus redoutable peut-être que l'enfermement dans un espace physique, l'intimité-enfermement enferme la parole dans le monologue et l'impossibilité de communiquer avec les autres. Dans le film comme dans le texte de Pinto, Ella entreprend plusieurs tentatives pour briser l'enfermement en racontant ses souffrances à d'autres : à des médecins, à des avocats (dans le récit littéraire), à un prêtre et à sa propre mère. Toutes ces tentatives se retournent finalement contre Ella, qui a l'impression de se heurter à un mur : en effet, $\hat{E} l$ est à la lettre un homme au-dessus de tout soupçon : il jouit d'une respectabilité sociale à toute épreuve, et, par ailleurs, est habile à travestir les faits.

La parole de Ella est rejetée a priori comme dépourvue de sens, ou bien comme le fruit d'une "imagination juvénile". « Je ne pouvais plus rien dire ! À quoi bon dire davantage ? » (Pinto, 2011a, p. 54) - écrit la narratrice, après une vaine tentative pour dire aux employés de maison présents qu'elle vient de subir une nouvelle agression : « mes phrases désespérées se heurtaient à l'apparence de vérité qui se dressait, fatalement et terriblement ironique audessus de ma tragédie » (Ibid.).

Cette expérience de l'inutilité ou de l'incapacité du langage traverse tout le récit de Mercedes Pinto : le prêtre comme le médecin, la mère comme les psychologues, tendent à rendre Ella coupable, et lui prescrivent toujours «...de la patience, de la résignation, de la douceur, de la modération » (Pinto, 2011a, p. 48). L'enfermement n'est pas seulement spatial : là où elle réussit à s'évader de la maison un moment pour tenter d'adresser un appel à l'aide à quelqu'un d'extérieur au couple, elle se découvre dans un enfermement plus profond encore, qui apparaît comme une forme de solitude radicale. Ella n'a personne au monde pour l'aider : sa propre mère rejette ses plaintes en lui demandant d'être plus douce et affectueuse envers Él. «No, no me digas nadareplicó atajándome -, pues, cuando un hombre habla tan con el alma y hasta llega a llorar, no puede mentir. Sé buena, trátalo con dulzura y la paz vendrá sobre vosotros » (Pinto, 2011a, p. 39). Ce dialogue est repris tel quel dans l'adaptation de Buñuel.

Cette forme d'enfermement-solitude ne peut être dissociée du contexte social dans lequel les deux œuvres ont été produites : pour le récit de Pinto, il s'agit de l'Espagne catholique, patriarcale et conservatrice des années 1920 (Pinto dut quitter l'Espagne en 1924, sous la dictature de Primo de Rivera, après avoir prononcé à l'université centrale de Madrid une conférence intitulée $D u$ divorce comme mesure d'hygiène). Pour le film de Buñuel (né en 1900), qui connut dans son enfance et sa jeunesse en Espagne une éducation catholique traditionaliste, il s'agit du Mexique post-révolutionnaire et postlazariste du milieu du XXe siècle, où l'ancienne aristocratie conservatrice - incarnée par Franciscoconserve encore une partie de ses privilèges et de son prestige social.

Dans les deux cas, il s'agit de sociétés où les traditions sociales et le traditionalisme catholique assignent à la femme un statut subalterne. La solitude éprouvée par la narratrice de Mercedes Pinto ou par Gloria se rattache aussi à des modèles normatifs qui assignent à la femme les rôles de la résignation et du sacrifice. L'idéologie du sacrifice nécessaire de la femme fut d'ailleurs, d'après Pinto, l'un des éléments à l'origine de son mariage avec l'homme qui deviendra le modèle de Él : Juan de Foronda y Cubillas, capitaine de la Marine marchande d'Espagne et professeur à l'École nautique des Iles Canaries.

Après le décès de son prétendant et le départ de sa sœur et confidente, Pinto éprouve une grande solitude et se tourne vers une religiosité « à la limite du fanatisme » : à un moment où dans son esprit «...gagnait du terrain l'idée obscure du sacrifice »(Pinto, 2001b, p. 220), elle décide d'épouser Él, à 
l'âge de 26 ans.

L'étonnante durée du couple Él- Ella (environ dix ans dans le récit Ella comme dans la biographie de Pinto) tient non seulement à la crainte de se voir séparée de ses enfants, mais aussi à un certain sentiment de pitié à l'égard d'un homme qu'elle considère malade.

\section{Une intimité à deux détruisant l'intimité du soi}

Construite comme intimité-enfermement, l'intimité à deux apparaît, dans les deux récits, comme une redoutable machine à détruire l'intimité personnelle de l'élément dominé - en l'occurrence, Ella. Cette destruction de l'intimité personnelle, qui est probablement la forme la plus caractérisée de la violence, s'effectue dans le récit de Pinto comme dans le film au moyen de deux dispositifs principaux : le contrôle de la subjectivité, d'une part et, d'autre part, la destruction du « monde » d'Ella, c'est-à-dire des choses qui ont pour elle du sens et de la valeur.

Le contrôle de la subjectivité d'Ella est une visée permanente de $E ́ l$. Sa forme la plus caractéristique est l'injonction à se dévoiler, à se rendre absolument transparente et disponible, à supprimer son intimité personnelle comme espace de la relation de soi à soi. « Dis-moi la vérité : à qui pensais-tu ? Parlemoi comme à ton confesseur : je suis ton mari » - dit Francisco à Gloria. L'analogie évoquée est singulièrement parlante : dans la pratique catholique de la confession - que Francisco, catholique pratiquant, est censé connaître -, celle-ci est un acte anonyme : elle se pratique normalement dans un confessionnal, qui est conçu de telle manière que le prêtre ne peut pas identifier la personne qui se confesse ; en d'autres termes, la confession, pratiquée dans les conditions règlementaires, préserve d'une certaine manière l'intimité personnelle du croyant. Or, la confession qu'exige Francisco passe outre la condition de l'anonymat, et, par là même, elle ne serait autre chose que l'invasion de la subjectivité de l'épouse dominée par le mari dominant. Comme toute invasion, l'invasion de la subjectivité est une prise de contrôle : le contrôle de la subjectivité d'Ella.

Par ailleurs, ce rapport de domination est déjà visible, de manière assez transparente, dans le choix de l'image du confesseur / mari (ou du mari-confesseur) : dans le rapport religieux entre le « confessant» et le confesseur il y a une asymétrie entre le premier, qui a le statut de pécheur et est demandeur d'absolution, et le second, qui seul dispose du pouvoir d'absoudre ou non ; dans le rapport épouse / époux, tel que le conçoit $E ́ l$, la première est fautive et doit rendre des comptes au second, qui seul détient le pouvoir de demander des comptes. Et la première faute de Ella est d'être Ella, c'est-à-dire d'être autre, d'avoir un vécu et une biographie singulière, bref, d'avoir un « monde » à elle. $E l \ll$ «..suivait tous mes pas, en poursuivant dans l'ombre mes pensées ; j'arrivai à avoir très peur (...) ; je ne me montrais jamais à la fenêtre, et je ne sortais au jardin qu'avec de grandes précautions » (Pinto, 2011a, p. 32).

La destruction du " monde » d'Ella est l'un des aspects les plus caractéristiques de l'intimitéenfermement construit par la relation asymétrique Él-Ella. Él détruit littéralement et physiquement les choses qui ont du sens et de la valeur pour Ella, et, à travers cette destruction, il vise Ella elle-même en tant que source autonome de sens et de valeurs. La destruction vise l'intimité personnelle d'Ella ou, si l'on préfère, la singularité d'Ella. Le récit de Pinto décrit assez précisément cette entreprise de destruction, qui touche tous les domaines de l'être, depuis l'être vivant jusqu'aux objets fabriqués par l'humain. Il y a d'abord la destruction des fleurs du jardin :

Le jardin m'attirait. (...). J'y trouvais de l'oubli, du repos, et mon âme semblait s'y rafraîchir dans un havre de paix (...). Or un après-midi Él découvrit ma retraite (...). Et le lendemain matin j'y trouvai toutes mes violettes arrachées. (Pinto, 2011a, p. 40-41).

Il y a ensuite la destruction d'un petit animal domestique :

On m'avait fait cadeau d'un chaton, et, dans ma solitude spirituelle, je lui portais une grande affection (...) [Or] sa haine à l'égard du petit chat atteint un tel point qu'un après-midi il le mit dans un sac et le jeta du haut de la terrasse (...). Par la suite, il mentit aux domestiques en leur disant que le chat "avait une maladie contagieuse" (Ibid., p. 32).

Puis il y a les humains proches d'Ella : non seulement Él interdit à Ella de voir ses anciennes connaissances et d'avoir ses propres amis, mais il tente aussi d'éloigner Ella de sa mère - non sans succès, comme nous l'avons vu -, et se montre hostile à la maternité d'Ella : «Quelle inopportunité ! »- lui lance-t-il lorsqu'elle lui apprend qu'elle est enceinte - «Avec tous les frais qu'on a ! " (Ibid., p. 20). Plus tard, quand les enfants seront plus grands - la narratrice d'Ella aura trois enfants avec $E$ l, de même que Mercedes Pinto -, Él s'efforcera de détruire la relation d'Ella avec ses enfants, comme en témoigne l'épisode du sapin de Noël installé par Ella : « Quand Él aperçut les cadeaux, il se mit en fureur, donna un coup de pied au sapin et le renversa, en hurlant : «Qu'est-ce que c'est que ces âneries»? ? (Ibid., p. 58).

\section{La personnalité autoritaire comme auto- enfermement}

Au terme de dix ans d'extrême souffrance, Ella réussit à s'évader de l'intimité conjugale violente qui l'enferme et détruit son intimité personnelle. Dans le récit de Mercedes Pinto, qui est tout à la fois une critique de l'hypocrisie morale de la société, un acte 
politique de dénonciation d'un système juridique et social qui assujettit la femme, et un mode d'expression qui permet à l'auteur d'" exorciser " (Cummings, 2006, p. 38) un traumatisme vécu, l'évasion est seulement signalée comme un fait du passé.

Elle intervient, comme dans la biographie de Pinto, au moment où Él, qui se trouvait enfermé depuis plusieurs mois dans un asile d'aliénés après une tentative de suicide, réussit à se faire libérer avec un faux certificat médical, par l'influence de sa famille. La narratrice décide alors de partir avec ses enfants et de quitter le pays : " Je fuis à travers les chemins de la vie et je ne savais pas vers où » (Pinto, 2011a, p. 91). À la différence du récit de Pinto, qui s'achève en laissant $E ́ l$ malade mais réintégré à la société, le récit de Buñuel propose une fin tout à fait différente : le film, qui est aujourd'hui considéré comme l'un des meilleurs du réalisateur espagnol, s'achève par une image d'enfermement : celle de Francisco, devenu moine dans un monastère en Colombie, après une crise de délire paranoïaque survenue dans une église de Mexico.

Dans la dernière séquence du film, Gloria et Raúl, devenus un couple marié avec un enfant, se trouvent de passage en Colombie et se rendent au monastère pour avoir des nouvelles de Francisco, qu'ils ne rencontrent pas. Le dernier plan de la séquence et du film montre Francisco face à un mur qu'il ne peut pas franchir - suggérant peut-être que la personnalité autoritaire, l'agent principal de la construction de l'intimité conjugale comme intimité-enfermement, est d'abord une personnalité enfermée.

\section{BIBLIOGRAPHIE}

$$
\text { Ramsay. }
$$

Buñuel, L. (2006). Mon dernier soupir. Paris: Éditions

Cummings Rendón, G. T. (2006). Buñuel y la novela hispana: un estudio de sus transposiciones mexicanas (Dissertation, Wayne State University, Michigan). [en ligne]. Michigan, Wayne State University. Disponible dans:http://proquest.umi.com/ pqdink? Ver $=1 \&$ Exp=06-06-2017\&FMT=7\&DID=1225119011\& $\mathrm{RQT}=309 \&$ attempt $=1 \& \mathrm{cfc}=1$

Pinto, M. (2011a). ÉL. Madrid: Ediciones Escalera.

Pinto, M. (2011b). Ella. Madrid: Ediciones Escalera. 